\title{
A Unique Presentation of Splenic Artery Aneurysm During Pregnancy
}

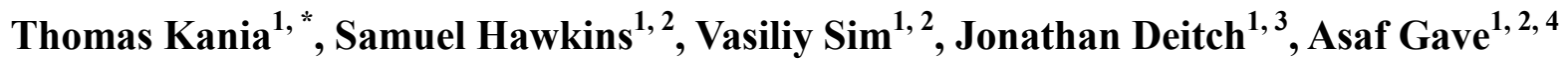 \\ ${ }^{1}$ Department of Surgery, Northwell Health, Staten Island University Hospital, New York, USA \\ ${ }^{2}$ Department of Trauma, Northwell Health, Staten Island University Hospital, New York, USA \\ ${ }^{3}$ Department of Vascular Surgery, Northwell Health, Staten Island University Hospital, New York, USA \\ ${ }^{4}$ Surgical ICU, Northwell Health, Staten Island University Hospital, New York, USA
}

Email address:

Tkania@northwell.edu (T. Kania), shawkins1@northwell.edu (S. Hawkins), vsim@northwell.edu (V. Sim), jdeitch@northwell.edu (J. Deitch), agave@northwell.edu (A. Gave)

${ }^{*}$ Corresponding author

\section{To cite this article:}

Thomas Kania, Samuel Hawkins, Vasiliy Sim, Jonathan Deitch, Asaf Gave. A Unique Presentation of Splenic Artery Aneurysm During Pregnancy. Journal of Surgery. Vol. 8, No. 4, 2020, pp. 114-117. doi: 10.11648/j.js.20200804.13

Received: April 27, 2020; Accepted: June 1, 2020; Published: June 16, 2020

\begin{abstract}
Visceral arterial (VAA) and, specifically, splenic arterial aneurysms (SAA) are rare entities often associated with pregnancy. When ruptured, they can be fatal and require emergent management. This case report includes a review of the relevant literature and describes a unique, multidisciplinary approach to managing such an aneurysm. We describe the difficult situation of an already rupturing aneurysm and the careful coordination of obstetric, surgical, vascular, and interventional radiology teams. The distinctiveness of this case continues with the intraoperative finding of metallic coils freely protruding into the peritoneum from a recently coil-embolized ruptured splenic artery aneurysm. Such iatrogenic foreign bodies are a product of the integration of multiple specialities that can provide life-saving care when managing critical surgical disease such as ruptured splenic artery aneurysms. Multidisciplinary approaches should be considered depending upon the resources available at one's institution.
\end{abstract}

Keywords: Splenic, Visceral, Abdominal, Arterial, Aneurysm, Pregnancy, Rupture, Coil, Embolizations

\section{Introduction}

Splenic artery aneurysms (SAA) are a rare but life-threatening entity, with a well-documented risk of rupture $[1,2]$. They are the third most common visceral aneurysm and are associated with states of high blood flow, such as pregnancy, AV fistula, and portal hypertension [3, 4]. Polyarteritis nodosa, endocarditis, and other inflammatory states can also serve as contributing factors. The most common pathophysiologic finding in visceral arterial aneurysm is a defect in tunica media [5].

These aneurysms commonly do not manifest until the sixth decade of life; in women of childbearing age, the prevalence of SAA was determined to be less than 0.1 percent $[6,7]$. However, when visceral arterial aneurysms do manifest in patients under 40 years of age, over 50 percent are pregnancy-related $[8,9]$.
There remains some debate as to optimal timing or size criteria regarding elective repair, and both open and endovascular techniques have shown to have good outcomes $[3,10-13]$. One fact is clear, however: ruptured splenic artery aneurysm, especially in the setting of pregnancy can be disastrous $[14,15]$.

\section{Case Presentation}

A 27-year-old female, gravida 2 para 1001 at 33 weeks, 4 days gestational age by last menstrual period, was brought in to the Emergency Department (ED) by EMS with a chief complaint of new onset, severe, chest, abdominal, and back pain at 02:00 in the morning. Her past medical history was pertinent only for a prior, term, uncomplicated spontaneous vaginal delivery. The patient stated that she was at work when she felt sudden onset of pain, accompanied by weakness, 
nausea, vomiting, and diaphoresis.

In the ED, she was alert and oriented, however, somnolent. At that time, she was hypotensive with an SBP of 50-60 $\mathrm{mmHg}$ and tachycardic to 140 beats per minute (BPM). On exam, the patient was pale, diaphoretic, and in distress, with a gravid abdomen that was diffusely tender. A bedside fetal ultrasound was performed, demonstrating fetal bradycardia to $60 \mathrm{BPM}$. The patient was given 2 liters of normal saline (NS) in the ED, then rushed to the Labor and Delivery Operating Room (L\&D OR) for an emergent Cesarean section.

In the L\&D OR, a Cesarean section through a Pfannensteil incision was performed. Upon peritoneal entry, 500cc of frank blood was evacuated from the abdomen. A live infant in vertex presentation was delivered; the infant was found to be floppy with no cry and was handed off to the Neonatal Intensive Care Unit (NICU) Team for resuscitation.

General surgery was consulted for ongoing intraperitoneal bleeding. A midline laparotomy was performed, large clots were evacuated, and all four quadrants were packed. Upon unpacking the left upper quadrant, large amounts of clots and active bleeding were noted, infiltrating the splenocolic ligament with hematoma. The short gastric arteries were taken down with a LigaSure vessel sealing device, the spleen and splenic flexure were both mobilized and a large $\sim 15 \mathrm{~cm}$ hematoma around the left kidney and retroperitoneum was visualized with an associated, palpable $5 \mathrm{~cm}$ pulsating mass.

The abdomen was once again packed, and the patient stabilized. Blood products were administered and an Abthera VAC temporary abdominal closure was placed. The patient was transported to the Post Anesthesia Care Unit (PACU) and then subsequently to the Interventional Radiology (IR) suite.

In IR, the left femoral artery was accessed, and a $2.8 \mathrm{Fr}$ microcatheter was used to select the splenic artery. An angiogram was performed, demonstrating a ruptured $6 \mathrm{~cm}$ splenic artery aneurysm within the midportion of the splenic artery (Figure 1). Multiple coils were utilized to embolize the splenic artery aneurysm as well as the inflow of the splenic artery. Repeat angiogram confirmed no flow through the aneurysm (Figure 2). The patient remained hemodynamically stable, and required no further blood products or vasopressors.

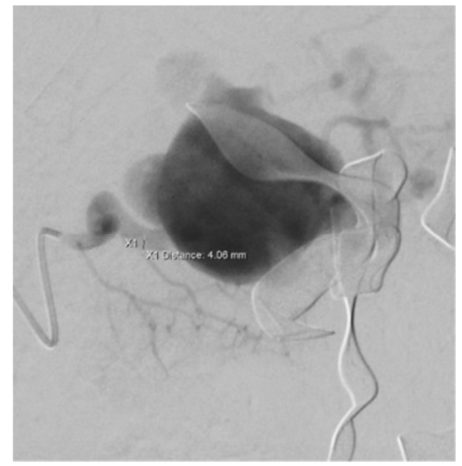

Figure 1. Initial angiogram demonstrating a $6 \mathrm{~cm}$ splenic artery aneurysm.

With the short gastric arteries transected and the splenic artery embolized, the patient was transported to the main OR for re-exploration, splenectomy, and closure, at which time a mass of coils were noted to be protruding from the aneurysm sac (Figure 3). Vascular surgery was called, and a decision was made to excise the aneurysm to prevent long-term migration of the coil into the peritoneal cavity. The tail of the pancreas and the splenic vessels were mobilized approximately $3 \mathrm{~cm}$ medial to the aneurysm and then transected en bloc with a vascular load EndoGIA stapler. The abdomen was irrigated, hemostasis was achieved, the abdomen was closed, and the patient was transferred to the PACU in stable condition.

The patient was monitored in the ICU, and remained hemodynamically stable. She was extubated on postoperative day 1 and discharged on postoperative day 4 with close follow up with both her obstetrician and surgeon, as well as with vaccination recommendations. Her child did not survive the initial resuscitation.

As an outpatient, a follow-up CT scan was performed which showed splenectomy, postsurgical changes, and no evidence of any pancreatic leak. The patient has since been seen as an outpatient in the office with no complications and is well.

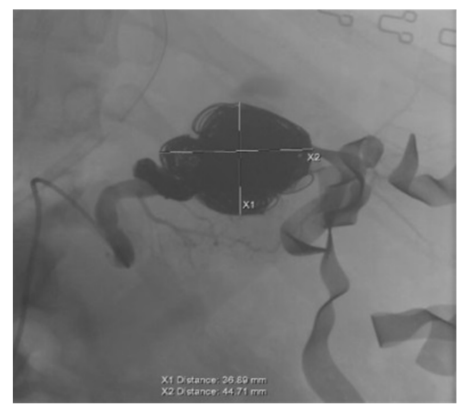

Figure 2. Completed coiling of splenic artery aneurysm along with repeat angiogram and measurements.

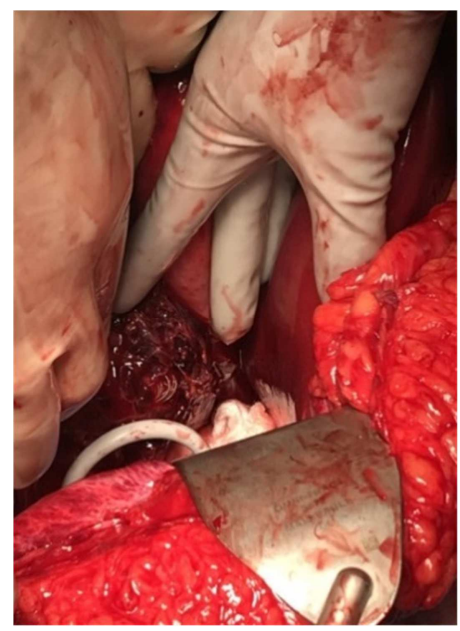

Figure 3. An intraoperative finding of a ruptured splenic artery aneurysm $s / p$ coil embolization, with coils protruding.

\section{Discussion}

This was a particularly challenging case requiring the coordination of multiple subspecialties including Gynecology, Neonatology, Interventional Radiology, General Surgery, and Vascular Surgery. The complexity of the case provides both opportunities for improvement and ongoing education. 
Presently, no routine screening for visceral artery aneurysms is recommended, and most are detected incidentally on imaging. The present lack of screening for this condition is unlikely to change given the rarity of these events as well as the risks of ionizing radiation in the setting of pregnancy [5]. Contrast enhanced ultrasonography so far has not proven to be reliable in diagnosis of splenic aneurysms [16, 17]. Treatment is recommended for patients with symptomatic, aneurysms greater than $2.5 \mathrm{~cm}$, pseudoaneurysms, portal hypertension, women of childbearing age or pregnancy [5].

In this patient with an aneurysm that has already ruptured, how should one proceed? Was IR intervention appropriate? The body of medical knowledge surrounding SAA and options for endovascular treatment stems largely from the trauma surgery literature. To date, there have been a number of reports describing the use of splenic artery embolization (SAE) either alone or in conjunction with surgery as part of a hybrid approach in hemodynamically stable patients [18-21]. This remains an area of ongoing research and further investigation is warranted, particularly in the setting of obstetric emergencies such as in this case [22-24].

For this patient, a hybrid approach was employed given the large $15 \mathrm{~cm}$ retroperitoneal hematoma that was obscuring the aneurysm itself. Dissection proximal to the aneurysm was attempted, however, due to the location of the aneurysm, it was apparent that if dissection continued, a distal pancreatectomy would have been necessary. This is in addition to the risk of ongoing massive hemorrhage in the setting of a retroperitoneal bleed that was both difficult to visualize and control. Given the patient's response to fluids and blood products, hemodynamic stability with packing, and the resources available, the intraoperative decision for embolization was made.

\section{Conclusion}

At institutions where interventional radiology is not available, operative management of ruptured SAA would therefore be the treatment of choice. For all others, there remains no consensus in the literature regarding the appropriate treatment for ruptured SAA, especially in the pregnant patient. Endovascular embolization has a lower perioperative morbidity compared with open surgery, but is usually reserved for smaller and non-ruptured aneurysms [25-27] For this patient, a multidisciplinary approach provided a favorable outcome in this worst-case-scenario of a large, proximal, ruptured splenic artery aneurysm in the setting of pregnancy.

\section{References}

[1] Hogendoorn W, Lavida A, Hunink MG, et al. Open repair, endovascular repair, and conservative management of true splenic artery aneurysms. J Vasc Surg. 2014; 60 (6): 1667-76. e1. doi: 10.1016/j.jvs.2014.08.067.

[2] Dave SP, Reis ED, Hossain A, Taub PJ, Kerstein MD, Hollier LH. Splenic artery aneurysm in the 1990s. Ann Vasc Surg. 2000; 14 (3): 223 - 229. doi: 10.1007/s100169910039.
[3] Berceli SA. Hepatic and splenic artery aneurysms. Semin Vasc Surg. 2005; 18 (4): 196-201.

[4] Yadav R, Tiwari MK, Mathur RM, Verma AK. Unusually giant splenic artery and vein aneurysm with arteriovenous fistula with hypersplenism in a nulliparous woman. Interact Cardiovasc Thorac Surg. 2009; 8 (3): 384-6.

[5] Ferreira RA, Ferreira MC, Ferreira DA, Ferreira AG, Ramos FO. Splenic artery aneurysm. Rev Col Bras Cir. 2016; 43 (5): 398-400.

[6] Pulli R, Dorigo W, Troisi N, Pratesi G, Innocenti AA, Pratesi C. Surgical treatment of visceral artery aneurysms: A 25-year experience. J Vasc Surg. 2008; 48 (2): 334-42.

[7] Abbas MA, Stone WM, Fowl RJ, Gloviczki P, Oldenburg WA, Pairolero PC, et al. Splenic artery aneurysms: two decades experience at Mayo clinic. Ann Vasc Surg. 2002; 16 (4): 442-9.

[8] McMahon DP, Ward WH, Harwood JL, Moore EM. An institutional review of splenic artery aneurysm in childbearing-aged females and splenic artery aneurysm rupture during pregnancy. Is screening justified? Mil Med. 2012; 177 (1): 96-8.

[9] Desai M, Wali AR, Birk HS, Santiago-Dieppa DR, Khalessi AA. Role of pregnancy and female sex steroids on aneurysm formation, growth, and rupture: a systematic review of the literature. Neurosurg Focus. 2019; 47 (1): E8. doi: 10.3171/2019.4.FOCUS19228.

[10] Habbal, Y, Christophi, C, Muralidharan, V. Aneurysms of the splenic artery $-\mathrm{a}$ review. Surgeon 2010; 8: 223-231. doi: 10.1016/j.surge.2009.11.011.

[11] Tessier, DJ, Stone, WM, Fowl, RJ. Clinical features and management of splenic artery aneurysm pseudoaneurysm: case series and cumulative review of literature. J Vasc Surg 2003; 38: 969-974. doi: 10.1016/S0741.

[12] Ouchi T, Kato N, Nakajima K, Higashigawa T, Hashimoto T, Chino S, Sakuma H. Splenic Artery Aneurysm Treated With Endovascular Stent Grafting: A Case Report and Review of Literature. Vasc Endovascular Surg. 2018 Nov; 52 (8): 663-668. doi: 10.1177/1538574418785252.

[13] Lakin RO, Bena JF, Sarac TP, et al. The contemporary management of splenic artery aneurysms. J Vasc Surg. 2011; 53 (4): 958-965. doi: 10.1016/j.jvs.2010.10.055.

[14] Lowry, Susan M., et al. "Splenic Artery Aneurysm Rupture." Obstetrics \& Gynecology, vol. 67, no. 2, 1986, pp. 291-292., doi: 10.1097/00006250-198602000-00026.

[15] Wiener Y, Tomashev R, Neeman O, et al. Splenic artery aneurysms during pregnancy: An obstetric nightmare. Eur J Obstet Gynecol Reprod Biol. 2019; 237: 121-125. doi: 10.1016/j.ejogrb.2019.04.029.

[16] Durkin N, Deganello A, Sellars ME, Sidhu PS, Davenport M, Makin E. Post-traumatic liver and splenic pseudoaneurysms in children: Diagnosis, management, and follow-up screening using contrast enhanced ultrasound (CEUS). J $\begin{array}{lllll}\text { Pediatr Surg. 2016; 51 } & \text { (2): } 289-292 \text {. doi: }\end{array}$ 10.1016/j.jpedsurg.2015.10.074.

[17] Cai DM, Parajuly SS, Ling WW, Li YZ, Luo Y. Diagnostic value of contrast enhanced ultrasound for splenic artery complications following acute pancreatitis. World J Gastroenterol. 2014; 20 (4): 1088-1094. doi: 10.3748/wjg.v20.i4.1088. 
[18] Bessoud B, Duchosal MA, Siegrist CA, et al. Prox- imal splenic artery embolization for blunt splenic injury: clinical, immunologic, and ultrasound- Doppler follow-up. J Trauma 2007; 62: 1481-1486.

[19] Haan JM, Biffl W, Knudson MM, et al. Splenic embolization revisited: a multicenter review. J Trauma 2004; 56: 542-547.

[20] Haan JM, Bochicchio GV, Kramer N, et al. Non- operative management of blunt splenic injury: a 5-year experience. J Trauma 2005; 58: 492-498.

[21] Igarashi Y, Ohki T, Maeda K, Kanaoka Y, Chono Y. Hybrid Surgery for Multiple Visceral Artery Aneurysms: A Case Report. Ann Vasc Surg. 2019; 60: 478. e19-.e24.

[22] Ahuja C, Farsad K, Chadha M. An Overview of Splenic Embolization. AJR Am J Roentgenol. 2015; 205 (4): 720-725. doi: 10.2214/AJR.15.14637.

[23] Ballout RA, Ghanem R, Nassar A, Hallal AH, Ghulmiyyah LM Splenic Artery Aneurysm (SAA) Rupture in Pregnancy: A Case Report of a Rare but Life-Threatening Obstetrical Complication. J Womens Health Dev. 2019; 2 (1): 19-27. doi: 10.26502/fjwhd.2644-2884004.
[24] Kashiura M, Yada N, Yamakawa K. Interventional radiology versus operative management for splenic injuries: a study protocol for a systematic review and meta-analysis. BMJ Open. 2019; 9 (8): e028172. Published 2019 Aug 10. doi: 10.1136/bmjopen-2018-028172.

[25] Björck M, Koelemay M, Acosta S, et al. Editor's Choice Management of the Diseases of Mesenteric Arteries and Veins: Clinical Practice Guidelines of the European Society of Vascular Surgery (ESVS). Eur J Vasc Endovasc Surg. 2017; 53 (4): 460-510. doi: 10.1016/j.ejvs.2017.01.010.

[26] Cordova AC, Sumpio BE. Visceral Artery Aneurysms and Pseudoaneurysms - Should They All be Managed by Endovascular Techniques?. Ann Vasc Dis. 2013; 6 (4): 687-693. doi: 10.3400/avd.ra.13-00045.

[27] Martinelli O, Giglio A, Irace L, Di Girolamo A, Gossetti B, Gattuso R. Single-Center Experience in the Treatment of Visceral Artery Aneurysms. Ann Vasc Surg. 2019; 60: 447-454. doi: 10.1016/j.avsg.2019.01.010. 